\title{
Identification and validation of distinct biological phenotypes in patients with acute respiratory distress syndrome by cluster analysis
}

\author{
L D Bos, ${ }^{1,2,3}$ L R Schouten, ${ }^{1,3}$ L A van Vught, ${ }_{1}^{4}$ M A Wiewel, ${ }_{1}^{4}$ D S Y Ong, ${ }_{1}^{5,6}$ O Cremer, ${ }_{1}^{6}$ \\ A Artigas, ${ }^{7}$ I Martin-Loeches, ${ }^{8}$ A J Hoogendijk ${ }^{4}$ T van der Poll, ${ }^{4}$ J Horn, ${ }^{1,3}$ \\ N Juffermans, ${ }^{1,3}$ C S Calfee, ${ }^{9}$ M J Schultz, ${ }^{1,3}$ On behalf of the MARS consortium
}

- Additional material is published online only. To view please visit the journal online (http://dx.doi.org/10.1136/ thoraxjnl-2016-209719).

For numbered affiliations see end of article.

Correspondence to Dr Lieuwe Bos, Department of Intensive Care, Academic Medical Center, Meibergdreef 9, Amsterdam 1105 AZ, The Netherlands; I.d.bos@amc.nl

Received 12 November 2016 Revised 27 March 2017 Accepted 28 March 2017 Published Online First 27 April 2017

\section{SLinked}

- http://dx.doi.org/10.1136/ thoraxjnl-2017-210422

\section{CrossMark}

To cite: Bos LD, Schouten LR, van Vught $L A$, et al. Thorax 2017;72:876-883.

\section{ABSTRACT}

Rationale We hypothesised that patients with acute respiratory distress syndrome (ARDS) can be clustered based on concentrations of plasma biomarkers and that the thereby identified biological phenotypes are associated with mortality.

Methods Consecutive patients with ARDS were included in this prospective observational cohort study. Cluster analysis of 20 biomarkers of inflammation, coagulation and endothelial activation provided the phenotypes in a training cohort, not taking any outcome data into account. Logistic regression with backward selection was used to select the most predictive biomarkers, and these predicted phenotypes were validated in a separate cohort. Multivariable logistic regression was used to quantify the independent association with mortality.

Results Two phenotypes were identified in 454 patients, which we named 'uninflamed' $(\mathrm{N}=218)$ and 'reactive' $(\mathrm{N}=236)$. A selection of four biomarkers (interleukin-6, interferon gamma, angiopoietin 1/2 and plasminogen activator inhibitor-1) could be used to accurately predict the phenotype in the training cohort (area under the receiver operating characteristics curve: $0.98,95 \% \mathrm{Cl} 0.97$ to 0.99 ). Mortality rates were $15.6 \%$ and $36.4 \%(p<0.001)$ in the training cohort and $13.6 \%$ and $37.5 \%(p<0.001)$ in the validation cohort $(\mathrm{N}=207)$. The 'reactive phenotype' was independent from confounders associated with intensive care unit mortality (training cohort: OR $1.13,95 \% \mathrm{Cl} 1.04$ to 1.23; validation cohort: $\mathrm{OR} 1.18,95 \% \mathrm{Cl} 1.06$ to 1.31 ). Conclusions Patients with ARDS can be clustered into two biological phenotypes, with different mortality rates. Four biomarkers can be used to predict the phenotype with high accuracy. The phenotypes were very similar to those found in cohorts derived from randomised controlled trials, and these results may improve patient selection for future clinical trials targeting host response in patients with ARDS.

\section{INTRODUCTION}

The acute respiratory distress syndrome (ARDS) is a major complication in critically ill patients, with high morbidity and mortality. ${ }^{1-4}$ Despite promising results in preclinical experiments testing immunomodulatory interventions in animals with lung injury, ${ }^{5}$ results from clinical trials in patients with

\section{Key messages}

What is the key question?

- Can cluster analysis of biological markers in plasma of patients with acute respiratory distress syndrome (ARDS) be used to identify phenotypes with different mortality rates?

What is the bottom line?

- There are at least two phenotypes of ARDS and the 'reactive' phenotype is associated with mortality independent of severity of illness.

\section{Why read on?}

- Pharmacological interventions in patients with ARDS have all failed so far; this study sheds light on two phenotypes of ARDS that may be targeted differently in future randomised controlled trials.

ARDS have been disappointing so far. ${ }^{7-9}$ Differences between pathological manifestations of lung injury in animals and ARDS in patients can only partly explain the discrepancies between animal studies and clinical trials. ${ }^{10}$ Furthermore, preclinical experiments have always used inbred animals in an effort to limit heterogeneity. Clinical trials, however, had to rely on clinical, radiological and physiological parameters to diagnose and stratify ARDS. ${ }^{11}$ Thus, patients with ARDS included in clinical trials are by definition more heterogeneous. ${ }^{12}$

Biological subtyping of patients could improve patient selection for clinical trials with targeted therapies, including immunomodulatory interventions, as has been shown in other pulmonary and non-pulmonary diseases. ${ }^{13}{ }^{14}$ Phenotyping of patients with ARDS can be done using clinical characteristics, causes of lung injury, ${ }^{15}$ individual or sets of biomarkers, ${ }^{16}$ or a combination of clinical and biological variables. ${ }^{17}$ Stratification on biological responses (ie, the biological phenotype) may allow for a better selection of patients, for example, with regard to potential benefit from a certain intervention (predictive enrichment), allowing exclusion of patients that have a low chance of benefit who may even may be harmed. ${ }^{18-20}$ Indeed, 
post hoc analyses of randomised controlled trials (RCTs) showed that a phenotype with increased inflammatory markers and decreased plasma bicarbonate levels is associated with the response to high positive end-expiratory pressure and restrictive fluid management. ${ }^{17} 21$

We hypothesised that patients with ARDS can be clustered based on plasma concentration of biomarkers of inflammation, coagulation and endothelial activation, and that these clusters would be associated with clinical outcomes. Importantly, the phenotypes are identified without taking any clinical data or outcomes into account. Additionally, we hypothesised that clusters could be discriminated based on a restricted number of plasma biomarkers, which would facilitate adoption of such phenotypes in future trials with the appropriate pharmacotherapy in patients with ARDS.

\section{METHODS}

\section{Study design}

This study was part of a biobank initiative called the 'Molecular Diagnosis and Risk Stratification for Sepsis' (MARS), a prospective cohort study performed in the intensive care units (ICUs) of two university-based tertiary care hospitals (the Academic Medical Center in Amsterdam and the University Medical Center Utrecht in Utrecht, both in the Netherlands) from 2011 to $2013^{8}$ (ClinicalTrials.gov identifier NCT01905033). The Institutional Review Boards of both hospitals approved the study protocol and opt-out consent method used for this study (IRB: 10-056C). The patients or their legal representatives were presented with a brochure and opt-out form, to be completed in case of unwillingness to participate.

\section{Setting}

Both ICUs are closed-format units, in which a team of boardcertified critical care physicians, fellows in critical care medicine and board-certified ICU nurses care for a mixed medical-surgical population of patients. The nurse to patient ratio was $1: 1$ to $1: 2$, depending on disease severity. Protocols that showed improved outcome of patients with ARDS were in place in both ICUs. This included lung-protective mechanical ventilation with low tidal volumes, ${ }^{22} 23$ higher positive end-expiratory pressure levels ${ }^{24}$ in patients with more severe forms of ARDS and prone positioning. ${ }^{25}$ Furthermore, a restrictive fluid protocol was used, ${ }^{26}$ and analgo-sedation was performed using validated sedation scales preferring bolus versus continuous sedation. ${ }^{27}$

\section{Inclusion and exclusion criteria}

Consecutive adult patients admitted to the ICU with an expected length of stay of $>24$ hours from January 2011 to June 2013 were eligible for participation in the MARS study. All patients with ARDS from whom plasma that was obtained on the day of diagnosis of ARDS was available in the biobank were eligible for the present study. Part of this cohort was described earlier, albeit with a different scientific question, and the definitions are equal to those used in that study. ${ }^{28}$ ARDS was defined according to the criteria stated by the American-European Consensus Conference on ARDS: ${ }^{29}$ that is, the diagnosis required an acute onset of symptoms, the presence of bilateral infiltrates on chest radiography, a pulmonary artery wedge pressure $<18 \mathrm{~mm} \mathrm{Hg}$ and/or the absence of signs of left ventricular dysfunction, and a $\mathrm{pO}_{2}$ in arterial blood to fraction of inspired oxygen ratio $\left(\mathrm{PaO}_{2} / \mathrm{FiO}_{2}\right) \leq 200$. Patients that met the above criteria but with a $\mathrm{PaO}_{2} / \mathrm{FiO}_{2} \leq 300$, classified as acute lung injury (ALI) according to the American-European consensus conference (AECC) criteria, were also included in the analysis since the updated definition of ARDS according to the 'Berlin' definition ${ }^{11}$ classifies this category as mild ARDS. Henceforth in this article patients with ALI according to AECC criteria are categorised as mild ARDS. Although our study started in 2011, before the recent 'Berlin' update of the ARDS definition was published, ${ }^{11}$ we found that no patients would have been excluded in case we had used the latest definition for ARDS. There were no additional inclusion or exclusion criteria.

\section{Diagnosis of ARDS}

A dedicated team of researchers who were regularly trained by studying case vignettes screened for the presence of ARDS on a daily basis while patients remained in the ICU. Patients were classified as having mild, moderate or severe ARDS according to the $\mathrm{PaO}_{2} / \mathrm{FiO}_{2}$ at the moment of diagnosis. Patients with ARDS were separated on the basis of having indirect (ie, systemic inflammatory response syndrome-associated or sepsis-associated ARDS) or direct ARDS (ie, pneumonia-associated, aspiration-associated or in association with another pulmonary cause).

\section{Training and validation cohort}

Patients that were included in the first 18 months of the study were included in the training cohort. The patients that were included in the subsequent year were used as a validation cohort.

\section{Blood sampling and biomarker assay}

Around day of ARDS diagnosis, blood was collected from all patients in a plastic vacuum container filled with EDTA. After centrifugation (1500G for $15 \mathrm{~min}$ ) plasma was frozen at $-80^{\circ} \mathrm{C}$ for batch-wise analysis. Interleukin (IL)-1 $\beta$, IL-6, IL-8, tumour necrosis factor $\alpha$, IL-10, IL-13, interferon gamma (IFN- $\gamma$ ), granulocyte macrophage-colony stimulating factor, soluble E-selectin, soluble p-selectin and fractalkine were measured in all samples with a cytometric bead array (CBA) Flex Set multiplex assay according to the instructions from the manufacturer (BD Biosciences, San Jose, California, USA). Plasminogen activator inhibitor (PAI)-1, D-dimer, tissue plasminogen activator, antithrombin, soluble intercellular adhesion molecule-1, matrix metalloproteinase-8, tissue inhibitor of metalloprotease 1, angiopoietin (ANG)1 and ANG2 were measured in all samples with Luminex according to the manufacturer instructions (BioRad, Hercules, California, USA). The values that were below the lower limit of quantification were set to the lowest value that we could accurately quantify. The upper limit of detection was not reached, with the exception of two markers (IFN- $\gamma$ and IL-10) in one sample; these values were set to the upper limit of quantification. These markers were selected to represent some of the major pathways that are associated with critical illness (see online supplementary table S1).

\section{Statistical analyses}

The analytical pipeline for analysis of biological clusters had six steps. First, biomarker data were $\log ^{10}$ transformed to obtain normally distributed variables, which was checked by Q-Q plots. Second, Ward clustering was performed with data from the patients in the training cohort only. Ward's method minimised the variance within a cluster and thereby selects patients with homogenous characteristics, in this case biomarker concentrations. Importantly, it does not take the clinical outcome of a patient into account. The optimal number of clusters was determined using the 'NbClust' package in R-statistics with the default settings. ${ }^{30}$ This algorithm combines 30 indices to deduce the optimal number of clusters in a given data set (see online 
supplement for more details). Third, the clusters were visualised using a heatmap and dendrogram with the 'gplots' package. Fourth, the clinical features of the clusters were compared and the independent association (corrected for Acute Physiology and Chronic Health Evaluation (APACHE IV) score, ${ }^{31}$ Age, $\mathrm{PaO}_{2}$ / $\mathrm{FiO}_{2}$ and Plateau pressure Score (APPS) ${ }^{32}$ and gender) of the biological cluster with mortality was studied by logistic regression. $\mathrm{PaO}_{2} / \mathrm{FiO}_{2}$ ratio and a direct cause for ARDS were studied as potential additional confounders. Missing clinical data were replaced using multiple imputation chained equations ${ }^{33}$ with the standard settings (five imputations; five iterations; see online supplementary table S2). Also, the orthogonality, or independent relation, of the biological clusters and the Berlin classification with regard to ICU mortality investigated by logistic regression were visualised with bar plots. Fifth, prediction of cluster membership was performed by multivariable logistic regression, with the 20 biomarkers as independent variables. Automated backward selection was used to identify the optimal set of predictors. This model was used to predict the phenotypes in the validation cohort. Routinely available clinical and biochemical variables were tested in the same way. Finally, step number six was a repetition of the methods described in step number four (eg, the independent association with ICU mortality) for patients that were included in the validation cohort. Importantly, the analyses in the validation cohort were completely independent from the training cohort.

A sensitivity analysis was performed in patients with a direct cause for ARDS and for patients without chronic respiratory failure and for hospital mortality instead of ICU mortality as the outcome of interest. The absolute change in the OR was evaluated to quantify sensitivity. Furthermore, the association between phenotype and steroid exposure in the ICU and timing of sample collection (day of diagnosis, day before or morning after) was evaluated with logistic regression.

Data were presented as median with IQR, mean with SD or absolute occurrence and percentage. Differences between groups were analysed by Mann-Whitney U, Kruskal-Wallis analysis of variance or $\chi^{2}$ test based on variable distribution and the number of groups. Receiver operating characteristics curve analysis was used to quantify the strength of discrimination. A p value $<0.05$ was used to determine statistical significance for all tests and 95\% CIs were given. All analyses were performed in $\mathrm{R}$ (V.3.2.2) using R-studio interface ( $\mathrm{R}$ Core Team. R: A Language and Environment for Statistical Computing. 2013. http://www.r-project.org/).

\section{RESULTS}

Included patients

Of 6994 consecutive admissions to the ICU, 873 patients met the criteria for ARDS during two and a half years in the two participating hospitals, of whom $700(80 \%)$ had complete biomarker data and were included in the present analysis. In total, 454 patients were included in the training cohort and 246 in the validation cohort. Seventy-three per cent of patients in the training cohort and $64 \%$ of the patients in the validation cohort came from hospital 1. Patient characteristics can be found in table 1. In total, $262(37 \%)$ patients had mild, 331 (47\%) had moderate and 107 (15\%) had severe ARDS. Also, 429 (61\%) patients had a pulmonary cause for ARDS. ICU mortality was $26 \%$.

\section{Identification of biological phenotypes}

Two clusters were separated based on plasma biomarkers of inflammation, coagulation and endothelial activation without taking the
Table 1 Patient characteristics, stratified for the training and validation cohort

\begin{tabular}{|c|c|c|c|}
\hline & $\begin{array}{l}\text { Training } \\
\mathrm{N}=454\end{array}$ & $\begin{array}{l}\text { Validation } \\
\mathrm{N}=246\end{array}$ & $p$ Value \\
\hline Age & $62(51-71)$ & $62(50-70)$ & 0.47 \\
\hline Male & $288(63.4)$ & $155(60)$ & 0.94 \\
\hline APACHE IV score & $83(63-104.8)$ & $82(62-108)$ & 0.91 \\
\hline \multicolumn{4}{|l|}{ Admission type } \\
\hline Medical & $310(68.3)$ & $188(76.4)$ & \multirow[t]{3}{*}{0.026} \\
\hline Planned surgical & $67(14.8)$ & $21(8.5)$ & \\
\hline Emergency surgery & 77 (17) & 37 (15) & \\
\hline \multicolumn{4}{|l|}{ Comorbidities } \\
\hline Diabetes mellitus & 66 (14.5) & $41(16.7)$ & 0.35 \\
\hline Alcohol abuse & $60(13.2)$ & $35(14.2)$ & 0.72 \\
\hline COPD & $44(9.7)$ & $25(10.2)$ & 0.90 \\
\hline Immune deficiency & $86(18.9)$ & $44(17.9)$ & 0.76 \\
\hline \multicolumn{4}{|l|}{ Risk factor* } \\
\hline Pneumonia & $256(56.4)$ & $144(58.5)$ & 0.63 \\
\hline Aspiration & $51(11.2)$ & $18(7.3)$ & 0.15 \\
\hline Other pulmonary & $4(0.9)$ & $0(0)$ & 0.31 \\
\hline Sepsis & $292(64.3)$ & $154(62.6)$ & 0.69 \\
\hline Trauma/surgery & $61(13.4)$ & $28(11.4)$ & 0.48 \\
\hline Pancreatitis & $11(2.4)$ & $5(2)$ & 0.78 \\
\hline Other non-pulmonary & $63(13.9)$ & $24(9.8)$ & 0.12 \\
\hline $\mathrm{PaO}_{2} / \mathrm{FiO}_{2}$ at diagnosis & $158(111-208)$ & $171(117-224)$ & 0.17 \\
\hline $\begin{array}{l}\mathrm{PaO}_{2} / \mathrm{FiO}_{2} 24 \text { hours after } \\
\text { diagnosis }\end{array}$ & 177 (134-233) & $192(141-247)$ & 0.07 \\
\hline PEEP at diagnosis & $10(7-13)$ & $10(8-12)$ & 0.09 \\
\hline PEEP 24 hours after diagnosis & $10(7-12)$ & $9(6-12)$ & 0.14 \\
\hline $\mathrm{P}_{\max }$ at diagnosis & $26(19-32)$ & $24(19-30)$ & 0.04 \\
\hline $\mathrm{P}_{\max } 24$ hours after diagnosis & $23(18-30)$ & $22(17-29)$ & 0.11 \\
\hline $\begin{array}{l}\text { Tidal volume/kg predicted } \\
\text { bodyweight }\end{array}$ & $7.1(6.2-8.2)$ & $7.2(6.4-8.6)$ & 0.19 \\
\hline APPS & $5(4-6)$ & $5(4-6)$ & 0.03 \\
\hline SOFA at diagnosis & $9(6-11)$ & $8(6-11)$ & 0.25 \\
\hline \multicolumn{4}{|l|}{ Berlin category } \\
\hline Mild & $153(33.7)$ & $109(44.3)$ & 0.015 \\
\hline Moderate & $231(50.9)$ & $100(40.7)$ & \\
\hline Severe & 70 (15.4) & 37 (15) & \\
\hline Days on mechanical ventilation & 7 (3-13) & $6(2-13)$ & 0.29 \\
\hline Days in the ICU & $8(4-15)$ & $8(4-15.8)$ & 0.99 \\
\hline Death in ICU & $120(26.4)$ & $64(26)$ & 0.93 \\
\hline 30 day mortality & $136(30)$ & 76 (30.9) & 0.86 \\
\hline \multicolumn{4}{|c|}{$\begin{array}{l}\text { Data are presented as the median with IQR for continuous variables and as number } \\
\text { with percentage for categorical variables. The } \mathrm{p} \text { value is calculated between the } \\
\text { training and validation cohort. Definitions for the variables are given in the definition } \\
\text { table at the end of the paper. } \\
\text { *Multiple risk factors per patient are possible. } \\
\text { APACHE, Acute Physiology and Chronic Health Evaluation; } \mathrm{APPS}, \mathrm{Age}_{1} \mathrm{PaO}_{2} / \mathrm{FiO}_{2} \text { and } \\
\text { Plateau pressure Score; ICU, intensive care unit; } \mathrm{MV} \text {, mechanical ventilation; } \mathrm{PEEP} \text {, } \\
\text { positive end expiratory pressure; SOFA, sepsis-related organ failure assessment. }\end{array}$} \\
\hline
\end{tabular}

clinical outcome into account (figure 1). Patients in cluster 1 were called to be from the 'uninflamed' phenotype. Patients in cluster 2 were called to be from the 'reactive' phenotype, with high plasma levels of markers of inflammation, coagulation and endothelial activation (see online supplementary figure $\mathrm{S} 1$ ).

\section{Association of phenotypes with clinical outcome in the training cohort}

Patients with a 'reactive' phenotype had a higher ICU mortality (36.4\%) compared with patients with an 'uninflamed' phenotype $(15.6 \%, \mathrm{p}<0.001)$. They also had higher APACHE IV 


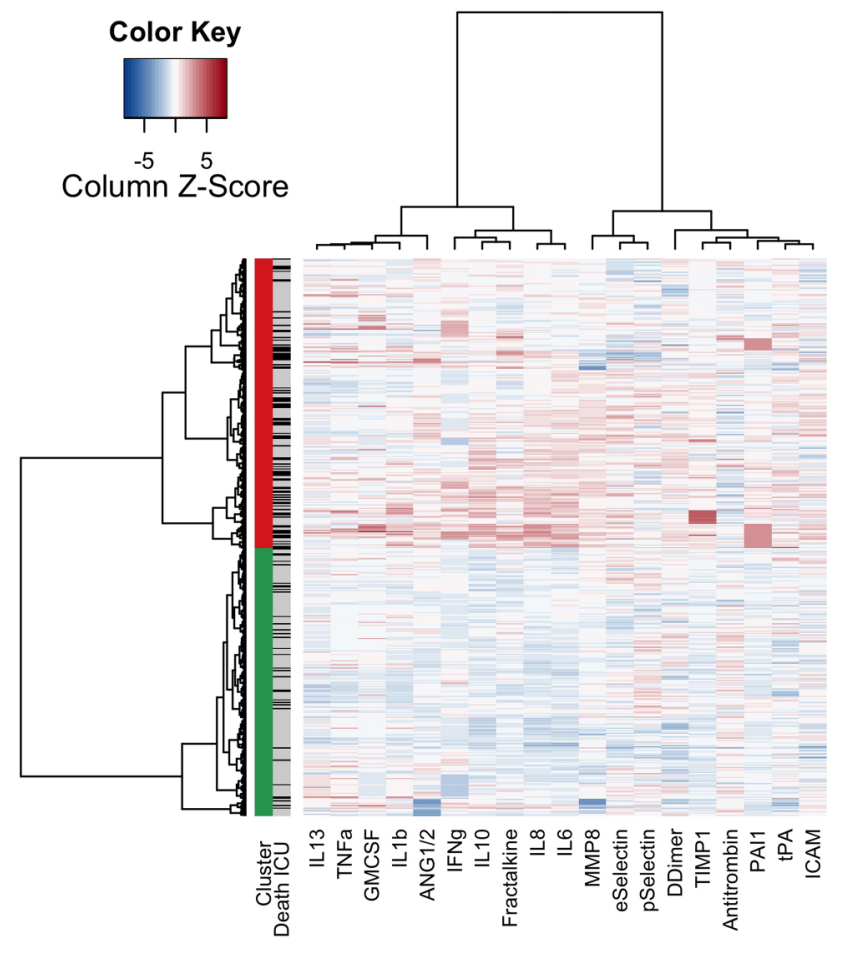

Figure 1 Heatmap of phenotypes. Columns: biomarkers. Rows: patients. First column: green blocks: 'uninflamed phenotype'; red: 'reactive phenotype'. Second column: patients that died are indicated with black, surviving patients with grey. Heat map: a higher concentration, in comparison to the other included patients is indicated with red, while a lower concentration is indicated by blue. ANG-1, angiopoietin-1; ICAM-1, intercellular adhesion molecule-1; ICU, intensive care unit; IFN, interferon; IL, interleukin; MMP8, matrix metalloproteinase-8; PAI-1, plasminogen activator inhibitor 1; TIMP1, tissue inhibitor of metalloprotease 1; TNF, tumour necrosis factor; tPA, tissue plasminogen activator.

scores, more organ failure and more frequently had an indirect cause for ARDS (table 2). A 'reactive' phenotype remained independently associated with ICU mortality after correction for APACHE IV (OR 1.13, 95\% CI 1.04 to 1.23). The addition of other potential confounders (APPS, $\mathrm{PaO}_{2} / \mathrm{FiO}_{2}$ ratio, pulmonary cause for ARDS) did not change this association (OR remained 1.11). The difference in mortality between the biological phenotypes was also independent of the Berlin classification of ARDS (figure 2, OR 3.1, 95\% CI 2.0 to 4.8 ).

\section{Prediction of phenotypes based on a limited set of biomarkers}

The plasma concentration of IL-6, IFN- $\gamma$, ANG1/2 and PAI-1 could be used to accurately discriminate between the two biological phenotypes in the training cohort (figure 3; area under the receiver operating characteristics curve: 0.98 , 95\% CI 0.97 to 0.99 ). The regression coefficients can be found in the online supplementary table S3. Prediction of the phenotype by routinely available variables that were significantly different between the phenotypes (APACHE IV, age, lactate, albumin, bicarbonate, mean arterial pressure, bicarbonate, platelets, $\mathrm{C}$ reactive protein, maximum inspiratory pressure, positive end expiratory pressure (PEEP) and $\mathrm{PaO}_{2} / \mathrm{FiO}_{2}$ ) had a significantly lower accuracy than that of the biomarkers (figure $3 ; \mathrm{p}<0.001$ ). The same discrimination could also be obtained by only using plasma albumin and bicarbonate concentration.
Table 2 Phenotypes versus clinical characteristics in training cohort

\begin{tabular}{|c|c|c|c|}
\hline & $\begin{array}{l}\text { Uninflamed } \\
\text { phenotype } \\
\mathrm{N}=218\end{array}$ & $\begin{array}{l}\text { Reactive } \\
\text { phenotype } \\
\mathrm{N}=236\end{array}$ & $p$ Value \\
\hline Age & $62(53.2-72)$ & $60(49-70)$ & 0.037 \\
\hline Male & $137(62.8)$ & $151(64)$ & 0.85 \\
\hline APACHE IV score & 69 (58-91) & $93(74-113)$ & $<0.001$ \\
\hline $\begin{array}{l}\text { APACHE IV acute } \\
\text { physiology score }\end{array}$ & $57(45-75)$ & $80(65-105)$ & $<0.001$ \\
\hline \multicolumn{4}{|l|}{ Admission type } \\
\hline Medical & $143(65.6)$ & $167(70.8)$ & 0.002 \\
\hline Elective surgery & $46(21.1)$ & $21(8.9)$ & \\
\hline Emergency surgery & $29(13.3)$ & $48(20.3)$ & \\
\hline Chronic renal insufficiency & $17(7.8)$ & $31(13.1)$ & 0.08 \\
\hline $\begin{array}{l}\text { Chronic respiratory } \\
\text { insufficiency }\end{array}$ & $24(11)$ & $8(3.4)$ & 0.002 \\
\hline COPD & $26(11.9)$ & $18(7.6)$ & 0.16 \\
\hline Diabetes mellitus & $33(15.1)$ & $33(14)$ & 0.80 \\
\hline Immune deficiency & $37(17)$ & $49(20.8)$ & 0.34 \\
\hline $\begin{array}{l}\text { Current drinking status } \\
\text { (alcohol) }\end{array}$ & $19(8.7)$ & $29(12.3)$ & 0.23 \\
\hline $\begin{array}{l}\text { Systemic corticosteroids } \\
\text { (before ICU) }\end{array}$ & $32(14.7)$ & $24(10.2)$ & 0.15 \\
\hline Direct hit for ARDS & $145(66.5)$ & $134(56.8)$ & 0.04 \\
\hline \multicolumn{4}{|l|}{ Berlin classification } \\
\hline Mild & 85 (39) & $68(28.8)$ & 0.07 \\
\hline Moderate & $103(47.2)$ & $128(54.2)$ & \\
\hline Severe & $30(13.8)$ & $40(16.9)$ & \\
\hline $\begin{array}{l}\text { Maximal inspiratory } \\
\text { pressure }\end{array}$ & $20(16-26)$ & $26(21-33)$ & $<0.001$ \\
\hline $\mathrm{PaO}_{2} / \mathrm{FiO}_{2}$ & $177.8(136-256)$ & $178(133-223)$ & 0.18 \\
\hline PEEP & $8(5-11)$ & $10(8-14)$ & $<0.001$ \\
\hline $\begin{array}{l}\text { Tidal volume/kg predicted } \\
\text { bodyweight }\end{array}$ & $7.1(6.3-8.1)$ & $7.1(6.2-8.3)$ & 0.92 \\
\hline APPS & $5(4-6)$ & $5(5-7)$ & 0.008 \\
\hline SOFA: circulation & $3(1-4)$ & $4(3-4)$ & $<0.001$ \\
\hline SOFA: CNS & $0(0-1)$ & $0(0-1)$ & 0.97 \\
\hline SOFA: coagulation & $0(0-1)$ & $1(0-2)$ & $<0.001$ \\
\hline SOFA: liver & $0(0-0)$ & $0(0-1)$ & $<0.001$ \\
\hline SOFA: renal & $0(0-1)$ & $1(0-3)$ & $<0.001$ \\
\hline SOFA: respiratory & $3(3-4)$ & $3(3-4)$ & 0.05 \\
\hline SOFA: total score & $7(5-9)$ & $10(8-12)$ & $<0.001$ \\
\hline $\begin{array}{l}\text { Days on mechanical } \\
\text { ventilation }\end{array}$ & $6(3-10)$ & $7(4-14.5)$ & 0.004 \\
\hline ICU length of stay & $7(4-12)$ & $10(5-19)$ & 0.006 \\
\hline Days free of MV at day 28 & $21(11-25)$ & $9(0-21)$ & $<0.001$ \\
\hline ICU mortality & $34(15.6)$ & $86(36.4)$ & $<0.001$ \\
\hline 30 day mortality & $47(21.6)$ & $89(37.7)$ & $<0.001$ \\
\hline
\end{tabular}

Data are presented as the median with IQR for continuous variables and as number with percentage for categorical variables. The $p$ value is calculated by the Kruskal-Wallis test for continuous variables and by Fisher's exact for categorical variables. Definitions for the variables are given in the definition table at the end of the paper.

APACHE, Acute Physiology and Chronic Health Evaluation; APPS, Age, $\mathrm{PaO}_{2} / \mathrm{FiO}_{2}$ and Plateau pressure Score; ARDS, acute respiratory distress syndrome; CNS, central nervous system; ICU, intensive care unit; MV, mechanical ventilation; PEEP, positive end expiratory pressure; SOFA, sepsis-related organ failure assessment.

\section{Association of phenotypes with clinical outcome in the validation cohort}

In the validation cohort, the predicted 'uninflamed' and 'reactive' phenotype had a mortality rate of $13.6 \%$ and $37.5 \%$ $(\mathrm{p}<0.001)$, respectively. The differences in clinical characteristics were comparable to those found in the training cohort 
Figure 2 Orthogonality of phenotypes and Berlin classification. Intensive care unit (ICU) mortality per phenotype and Berlin classification. Boxes indicate phenotypes and the training or validation cohort, separate bars Berlin categories. Differences in mortality between the 'reactive' phenotype and 'uninflamed' phenotype were independent of the Berlin classification of acute respiratory to 4.8 ) in the training cohort and in the validation cohort (OR $3.8,95 \% \mathrm{Cl}$ 2.0 to 7.2 ). distress syndrome (OR 3.1, 95\% Cl 2.0

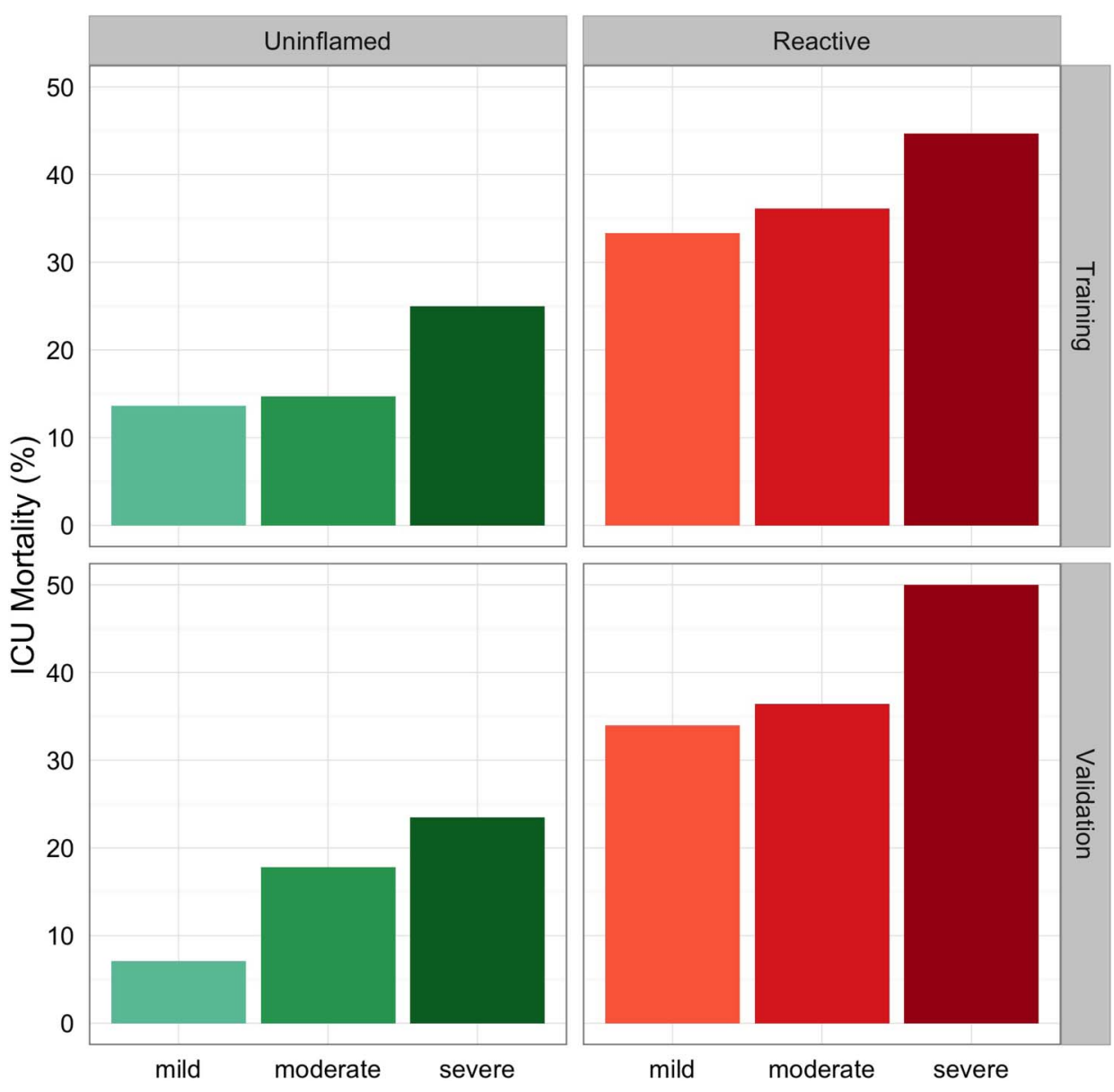

(online supplementary table S4, figures S3 and S4). A 'reactive' phenotype was independently associated with ICU mortality after correction for APACHE IV (OR 1.18, 95\% CI 1.06 to 1.31) and for the Berlin classification for severity of ARDS (OR $3.8,95 \%$ CI 2.0 to 7.2 ).

\section{Confounding factors and association of phenotype with hospital mortality}

Patients in whom the sample was taken the day before or after the diagnosis of ARDS were not more or less likely to be classified as having an 'uninflamed' or 'reactive' phenotype $(p=0.34$ for sample taken before and $p=0.13$ for taken after). A sensitivity analysis for patients with a pulmonary cause for ARDS alone and for patients without chronic respiratory failure also showed no change in OR. Sensitivity analysis on the association between the phenotypes and hospital mortality showed similar results as in the primary analysis (OR 1.10, 95\% CI 1.03 to 1.18). Exposure to steroids on the ICU (147/700, 21\%) was associated with a higher likelihood of a 'reactive' phenotype (OR 2.1, 95\% CI 1.5 to 3.1 ).

\section{DISCUSSION}

Two biologically distinct clusters of patients with ARDS could be identified. Outcome data were not taken into account when separating these clusters. Patients with the 'reactive' phenotype were approximately twice as likely to die during their stay in the ICU. Importantly, the biological phenotypes contained additional information compared with two mortality prediction scores and the Berlin classification for ARDS, and these results were validated in an independent group of patients. A 'reactive' phenotype could be predicted with the plasma concentration of four biomarkers and routinely available variables led to a less accurate prediction. We speculate that these biological phenotypes might be used to include patients for the appropriate pharmacological therapy in clinical trials.

To our knowledge, this is the first study to cluster patients with ARDS based on biomarker concentrations alone. Cluster analysis maximises the differences between patients, without taking the clinical outcome of a patient into account. Therefore, it is very different from, for example, logistic regression with single biomarkers. ${ }^{34}$ Calfee et al previously showed two distinct clusters of patients with ARDS within the cohorts of two large clinical trials. ${ }^{17}$ Both clinical and biomarker data were used to cluster the patients, and the clusters responded differently to randomly allocated changes in ventilator settings. Interestingly, mortality in the phenotypes that we identified was similar to that found in the phenotypes in their study (eg, $\pm 20 \%$ vs $\pm 45 \%)$. The phenotypes found in the Calfee study were replicated in another RCT population, in which the influence of fluid resuscitation management was tested. ${ }^{21}$ That study also revealed similar mortality rates and found that the response to randomly allocated fluid management differed per phenotype. All three studies found an increase in plasma IL- 8 and PAI- 1 concentration and a decrease in bicarbonate concentration in the 'reactive' or 'hyperinflammatory' phenotype. Therefore, we can speculate that the identified phenotypes could be the same between this observational study and the three RCTs, even though the prevalence of a 'reactive' phenotype is higher in our study. This finding would be notable because of the differences between the studies; observation and interventional, recruitment on 
Figure 3 Discrimination of biological phenotype based on a limited set of biomarkers in the training cohort. for the biological phenotype based on (1) biomarkers depicted in black: plasma concentrations of interleukin- 6 , interferon- $\gamma$, angiopoietin-1/2 and plasminogen activator inhibitor-1 (see online supplementary table S3) and (2) routinely available clinical variables depicted in grey; the same accuracy could be obtained with albumin and bicarbonate only. AUC, area under the curve. Receiver operating characteristics curve

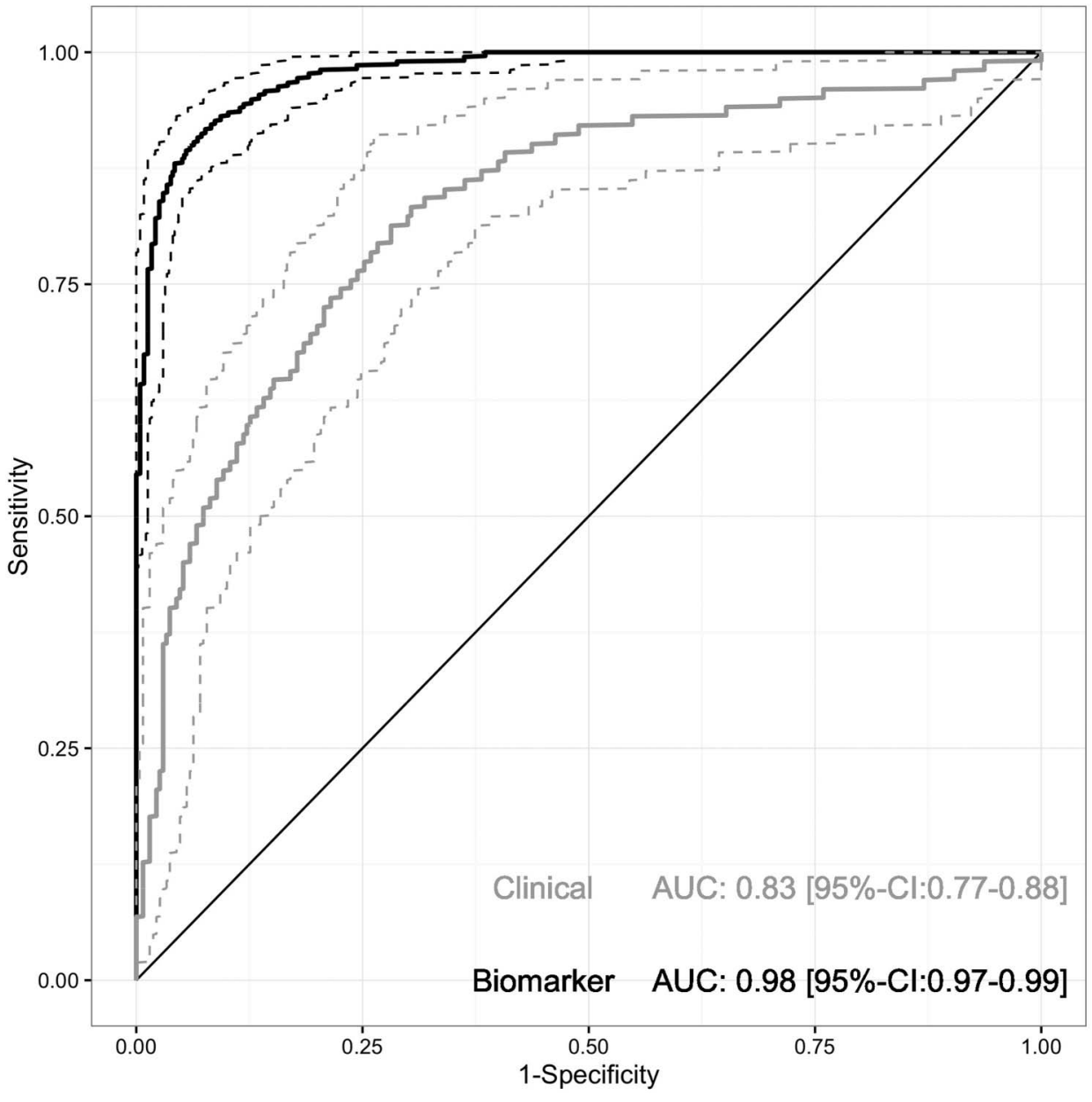

different continents and inclusion periods spanning $>15$ years between the ARMA trial and this study. Furthermore, the Calfee et al and Famous et al studies used both clinical and biomarkers data while we limited the analysis to biomarker data alone. Finally, the studies used different methods of clustering. The fact that the results show the identification of very similar phenotypes suggests the underlying identified biological signal is very strong.

The 'reactive' phenotype had a higher ICU mortality and might be used to select more severely ill patients for clinical trials (prognostic enrichment). Importantly, this association was independent of APACHE IV score, a frequently used validated and repeatedly calibrated prognostic score for mortality in the ICU. There was also added value of the biological clusters to the Berlin definition in the prediction of mortality. The results imply that the 'reactive' phenotype is not just a more severe form of ARDS and most definitely not captures the same grades of severity as the Berlin classification. This finding also illustrates that phenotypic clustering provides additional information, on top of the more frequently used clinical and physiological information. The $\mathrm{PaO}_{2} / \mathrm{FiO}_{2}$ is, until now, the only characteristic that has been used for phenotypic inclusion into clinical trials with patients with ARDS, ${ }^{24} 25^{35}$ with moderate success. Several interventions had positive effects on mortality in a selected group of ARDS with a low $\mathrm{PaO}_{2} / \mathrm{FiO}_{2}$ ratio. ${ }^{24}{ }^{25} \quad 35$ Interestingly, these were all interventions that aimed for physiological changes to improve oxygenation. In stark contrast, pharmacological interventions aimed at the immune system have repeatedly showed no benefit when applied to unselected ARDS patient groups. $^{7-9}$
We speculate that the phenotypes, derived from biological data alone, as identified in this study could be used to target pharmacological interventions to those patients that benefit most in future clinical trials. Improved patient selection and targeted intervention is the premise of this so-called 'stratified medicine' or 'precision medicine'. ${ }^{36}$ The efficacy of some pharmacotherapies could potentially be improved by correctly selecting the subgroups of patients that show molecular signs of susceptibility (predictive enrichment). Simultaneously, this approach may limit exposure of patients that would not benefit, but would have side effects. Thus, stratified medicine may increase efficiency of a drug in two ways: increase benefit and decrease harm. As for the phenotypes identified in this study, we postulate that the 'reactive' phenotype might benefit most from immunomodulatory interventions, such as corticosteroids, macrolides or others. On the other hand, the 'uninflamed' phenotype may be less likely to benefit from these approaches and/or may be more likely to be harmed, as mortality is infrequent and there is little inflammatory response.

ARDS phenotyping could allow for a more targeted pharmacological intervention in clinical trials and, if shown to be beneficial, in clinical practice. There are, however, several prerequisites before that hypothesis can be tested. The first, prediction of cluster membership by a minimal number of biomarkers, is explored in this paper. IL-6, IFN- $\gamma$, ANG1/2 and PAI- 1 concentrations in plasma drawn at the moment of ARDS diagnosis were sufficient to discriminate between patients with and without a 'reactive' phenotype. A four-biomarker assay is sufficiently small to allow for phenotyping of patients in clinical 
trials and clinical practice and clinical data alone do not discriminate sufficiently. A second prerequisite is rapid analyses of these biomarkers. We used CBA and Luminex to quantify the biomarker concentrations in this study. These methods are very suitable for batch-wise analysis in observational studies but would be very impractical to select patients for inclusion into clinical trials. Ideally, the result of the test would be available within several hours so that pharmacological therapy can be commenced early in the disease course.

Several limitations and strengths to our study are noticeable. First, we investigated the biological clusters only by means of a limited number of plasma biomarkers. It could be argued that we are still missing several relevant biomarkers that would disclose additional phenotypes. For example, there have been reports on soluble thrombomodulin, ${ }^{37}$ high-mobility group box 1 protein and sRAGE. ${ }^{38}$ However, even though we chose to restrict our analyses to a limited set of only 20 biomarkers, these biomarkers do represent three major pathways involved in lung injury. ${ }^{39}$ We cannot exclude that the addition of other markers would provide us with different phenotypes. The same point can be made for the size of the clusters. Therefore, we do not claim that two is the definitive number of phenotypes for ARDS, but we suggest that there are at least two phenotypes and that this should be taken into account in future research. Additionally, we were unable to associate phenotype with treatment response. This is due to the observational nature of our study. There would be additional value for identification of these phenotypes in patients that were included into RCTs that tested a pharmacological intervention targeting the immune system.

The observational nature of this study is also one of its strengths as it allowed for the inclusion of all consecutive patients with ARDS during 3 years in two university hospitals, and the results are therefore more likely representing the population as encountered on the ICU in daily practice. This is illustrated by the representative prevalence of ARDS, around $10 \%$ of ICU patients, ${ }^{2} 4$ although the ICU mortality was lower than in the LUNG SAFE study. ${ }^{2}$ The results were validated in a cohort that was separated over time; future studies should focus on replicating these results in an entirely independent cohort. Because biomarkers and clinical characteristics were separated completely in the analysis, we could determine that there is considerable orthogonality between the biological response within the patient and the clinical condition as observed from outside. This exemplifies that it is unpractical and may even be impossible to predict the phenotypes by clinical variables alone, as was also shown in the recent study by Famous et al. ${ }^{21}$ Finally, the physicians treating the patients were completely blind for the results of the biomarker assay as they were analysed only after completion of the study. Thereby, this study is not biased by 'self-fulfilling prophecies'; clinical parameters that are frequently used for mortality prediction or phenotyping are also available to the treating clinician who may consciously or unconsciously adapt treatment strategies based on this information, which in turn may influence clinical outcome or the association between the variable and outcome.

In conclusion, two biologically distinct clusters of patients with ARDS could be identified with a set of 20 biomarkers of inflammation, coagulation and endothelial activation. Mortality was double in the 'reactive' phenotype group than in the 'uninflamed' phenotype group. A 'reactive' phenotype could be predicted with the plasma concentration of four biomarkers and was independently associated with mortality, also in a validation cohort. These results may improve patient selection for future clinical trials targeting the immune system in patients with ARDS.

\section{DEFINITIONS}

\begin{tabular}{|c|c|}
\hline Term & Definition \\
\hline Chronic renal insufficiency & Chronically increased plasma creatinine before ICU admission or renal replacement therapy. \\
\hline $\begin{array}{l}\text { Chronic respiratory } \\
\text { insufficiency }\end{array}$ & Marked limitations in physical activity due to chronic respiratory disease or home oxygen support. \\
\hline Cluster & A naturally occurring subgroup of a population. \\
\hline COPD & History of COPD in medical records or anamnestic. \\
\hline $\begin{array}{l}\text { Current drinking status } \\
\text { (alcohol) }\end{array}$ & Anamnestic daily alcohol consumption or alcohol dependence. \\
\hline Diabetes mellitus & History of diabetes mellitus in medical records or anamnestic. \\
\hline Direct hit for ARDS & Pulmonary causative factor for ARDS. Pneumonia, aspiration, smoke inhalation or near drowning. \\
\hline Endotype & $\begin{array}{l}\text { A subset of patients defined by a distinct functional or pathobiological mechanism. Endotypes often confer both a differential risk of } \\
\text { disease-related outcome and a differential response to a therapy. Thus, such a markers may enable both predictive and prognostic } \\
\text { enrichment. }\end{array}$ \\
\hline Immune deficiency & Inherited or acquired immune deficiency (eg, HIV) or the usage of immunosuppressive medication (eg, chemotherapy). \\
\hline Observed phenotype & The phenotype (see definition) that is observed in all collected data through cluster analysis. \\
\hline Personalised medicine & The tailoring of medical treatment to the individual unique characteristics of each patient. ${ }^{20}$ \\
\hline Phenotype & $\begin{array}{l}\text { A clinical entity defined by observable characteristics that are produced by interactions of the genotype and the environment. The term is often } \\
\text { used to describe subsets based on clinical or biochemical variables, natural history, manifestations of disease and/or response to treatment } \\
\text { without any implication about mechanism. } 40\end{array}$ \\
\hline Prognostic enrichment & Identifying and focusing on high-risk patients. \\
\hline Predictive enrichment & Identifying and focusing on patients who are more likely to respond to the therapy being studied. \\
\hline Predicted phenotype & The phenotype (see definition) that is predicted by a subset of the markers that was used for phenotype discovery. \\
\hline Stratified medicine & $\begin{array}{l}\text { The tailoring of medical treatment to the individual characteristics of each patient. It does not literally mean the creation of drugs or medical } \\
\text { devices that are unique to a patient, but rather the ability to classify individuals into stratified subpopulations that differ in their susceptibility } \\
\text { to (or severity of) a particular disease or their response to a specific treatment. Preventive or therapeutic interventions can then be } \\
\text { concentrated on those who will benefit, sparing expense and side effects for those who will not. }\end{array}$ \\
\hline $\begin{array}{l}\text { Systemic corticosteroids } \\
\text { (before ICU) }\end{array}$ & Administration of systemic corticosteroids before admission to the ICU. \\
\hline
\end{tabular}




\section{Author affiliations}

'Department of Intensive Care, Academic Medical Center, Amsterdam, The Netherlands

${ }^{2}$ Department of Respiratory Medicine, Academic Medical Center, Amsterdam, The Netherlands

${ }^{3}$ Laboratory of Experimental Intensive Care and Anesthesiology (L.E.I.C.A), Academic Medical Center, Amsterdam, The Netherlands

${ }^{4}$ Center for Experimental and Molecular Medicine (CEMM), Academic Medical Center, Amsterdam, The Netherlands

${ }^{5}$ Department of Medical Microbiology, University Medical Center Utrecht, Utrecht,

The Netherlands

${ }^{6}$ Department of Intensive Care Medicine, University Medical Center Utrecht, Utrecht, The Netherlands

${ }^{7}$ CIBER enfermedades respiratorias (CIBERES), Critical Care Center, Sabadell Hospital, Corporación Sanitaria Universitaria Parc Taulí, Universitat Autonoma de Barcelona Sabadell, Spain

${ }^{8}$ Multidisciplinary Intensive Care Research Organization (MICRO), Department of Clinical Medicine, Trinity Centre for Health Sciences, Dublin, Ireland

${ }^{9}$ Departments of Medicine and Anesthesia, Cardiovascular Research Institute, University of California San Francisco, San Francisco, California, USA

Acknowledgements The authors sincerely thank everyone involved in the MARS project. More information on this public, private consortium can be found on http:// www.ctmm.nl/en/projecten/infectie/mars? set_language $=$ en. LB had full access to all of the data in the study and takes responsibility for the integrity of the data and the accuracy of the data analysis.

Collaborators MARS Consortium are Jos F Frencken, (Department of Intensive Care Medicine and Julius Center for Health Sciences and Primary Care, University Medical Center Utrecht, Utrecht, the Netherlands); Marc Bonten, Peter M C Klein Klouwenberg, David Ong (Department of Medical Microbiology, University Medical Center Utrecht, Utrecht, the Netherlands) and Roosmarijn T M van Hooijdonk, Mischa A Huson, Laura R A Schouten, Marleen Straat, Lonneke A van Vught, Maryse A Wiewel, Esther Witteveen, Gerie J Glas, and Luuk Wieske, (Department of Intensive Care Medicine, Academic Medical Center, University of Amsterdam); Brendon P Scicluna, Arjan J Hoogendijk, H Belkasim-Bohoudi, Tom van der Poll (Center of Experimental Molecular Medicine; CEMM, Academic Medical Center, University of Amsterdam). The list of MARS Consortium members is also available as an online supplementary appendix.

Contributors LDB, LRS and MJS: study design, data collection, data analysis, data interpretation and writing. LAV, MAW, DO OC and AJH: data collection, data analysis, data interpretation and writing. $\mathrm{AA}$, IML, TvdP, JH and NF: study design, data analysis, data interpretation and writing. CSC: data analysis, data interpretation and writing.

Funding Center for Translational Molecular Medicine, 10.13039/501100006020, MARS project.

Competing interests CC was supported by the NIH (HL133390 and HL131621).

Ethics approval UMCU and AMC (IRBNo.10-056C).

Provenance and peer review Not commissioned; externally peer reviewed.

\section{REFERENCES}

1 Hudson LD, Steinberg KP. Epidemiology of acute lung injury and ARDS*. Chest 1999:116:74S-82S

2 Bersten $A D$, Edibam C, Hunt $T$, et al. Incidence and mortality of acute lung Injury and the Acute Respiratory Distress Syndrome in Three Australian States. Am J Respir Crit Care Med 2002:165:443-8.

3 Rubenfeld GD, Caldwell E, Peabody E, et al. Incidence and outcomes of acute lung injury. N Engl J Med 2005;353:1685-93.

4 Gattinoni L, Haren F, Van Larsson A, et al. Epidemiology, patterns of care, and mortality for patients with acute respiratory distress syndrome in intensive care units in 50 countries. JAMA 2016;315:788-800.

5 Calfee CS, Matthay MA. Nonventilatory treatments for acute lung injury and ARDS. Chest 2007;131:913-20.

6 Beitler JR, Schoenfeld DA, Thompson BT. Preventing ARDS: progress, promise, and pitfalls. Chest 2014;146:1102-13.

7 Takeda S, Ishizaka A, Fujino Y, et al. Time to change diagnostic criteria of ARDS: toward the disease entity-based subgrouping. Pulm Pharmacol Ther 2005;18:115-9.

8 Boyle AJ, McNamee JJ, McAuley DF. Biological therapies in the acute respiratory distress syndrome. Expert Opin Biol Ther 2014;14:969-81.

9 Cepkova M, Matthay MA. Pharmacotherapy of acute lung injury and the acute respiratory distress syndrome. J Intensive Care Med 2006;21:119-43.

10 Matute-Bello G, Downey G, Moore BB, et al. An official American Thoracic Society workshop report: features and measurements of experimental acute lung injury in animals. Am J Respir Cell Mol Biol 2011;44:725-38.

11 Ranieri VM, Rubenfeld GD, Thompson BT, et al., ARDS Definition Task Force. Acute respiratory distress syndrome: the Berlin definition. JAMA 2012;307:2526-33.

12 Thille AW, Esteban A, Fernández-Segoviano P, et al. Comparison of the Berlin definition for acute respiratory distress syndrome with autopsy. Am I Respir Crit Care Med 2013;187:761-7.
13 Durham AL, Caramori G, Chung KF, et al. Targeted anti-inflammatory therapeutics in asthma and chronic obstructive lung disease. Trans/ Res 2015;167:192-203.

14 Wood SL, Pernemalm M, Crosbie PA, et al. Molecular histology of lung cancer: from targets to treatments. Cancer Treat Rev 2015:41:361-75.

15 Agarwal R. Is the mortality higher in the pulmonary vs the extrapulmonary ARDS? * CHEST I 2008:133:1463.

16 Calfee CS, Janz DR, Bernard GR, et al. Distinct molecular phenotypes of direct versus indirect ARDS in single and multi-center studies. Chest 2015;147:1539-48.

17 Calfee CS, Delucchi K, Parsons PE, et al. Subphenotypes in acute respiratory distress syndrome: latent class analysis of data from two randomised controlled trials. Lancet Respir Med 2014;2:611-20.

18 Rittirsch D, Flierl MA, Ward PA. Harmful molecular mechanisms in sepsis. Nat Rev Immunol 2008:8:776-87.

19 Schulzer M, Mancini GB. 'Unqualified success' and 'unmitigated failure': number-needed-to-treat-related concepts for assessing treatment efficacy in the presence of treatment-induced adverse events. Int I Epidemiol 1996;25:704-12

20 WHO. Stratified medicine and pharmacogenomics. Prior Med Eur World Updat Rep 2013;4:1-5. http://www.who.int/medicines/areas/priority_medicines/Ch7_4Stratified.pdf

21 Famous KR, Delucchi K, Ware LB, et al. Acute respiratory distress syndrome subphenotypes respond differently to randomized fluid management strategy. Am J Respir Crit Care Med 2017:195:331-8.

22 ARDS-Network. Ventilation with lower tidal volumes as compared with traditional tidal volumes for acute lung injury and the acute respiratory distress syndrome. The Acute Respiratory Distress Syndrome Network. N Engl J Med 2000:342:1301-8.

23 Putensen C, Theuerkauf N. Meta-analysis: ventilation Strategies and outcomes of the acute respiratory distress syndrome and acute lung injury. Ann Intern Med 2013;151:566-76

24 Briel M, Meade M, Mercat A, et al. Higher vs lower positive end-expiratory pressure in patients with acute lung injury and acute respiratory distress syndrome: systematic review and meta-analysis. JAMA 2010;303:865-73.

25 Hu SL, He HL, Pan C, et al. The effect of prone positioning on mortality in patients with acute respiratory distress syndrome: a meta-analysis of randomized controlled trials. Crit Care 2014:18:R109.

26 Wiedemann HP, Wheeler AP, Bernard GR, et al., National Heart and Blood Institute Acute Respiratory Distress Syndrome (ARDS) Clinical Trials Network. Comparison of two fluid-management strategies in acute lung injury. N Eng/ J Med 2006;354:2564-75.

27 Minhas MA, Velasquez AG, Kaul A, et al. Effect of protocolized sedation on clinical outcomes in mechanically ventilated intensive care unit patients: a systematic review and meta-analysis of randomized controlled trials. Mayo Clin Proc 2015;90:613-23.

28 Geboers DGPJ, de Beer FM, Tuip-de Boer AMT, et al. Plasma suPAR as a prognostic biological marker for ICU mortality in ARDS patients. Intensive Care Med 2015:41:1281-90.

29 Bernard GR, Artigas A, Brigham KL, et al. The American-European Consensus Conference on ARDS. Definitions, mechanisms, relevant outcomes, and clinical trial coordination. Am J Respir Crit Care Med 1994;149:818-24. http://www.ncbi.nlm.nih. gov/entrez/query.fcgi?cmd=Retrieve\&db=PubMed\&dopt=Citation\&list_uids=7509706

30 Charrad M, Ghazzali N, Boiteau V, et al. NbClust : an R Package for determining the relevant number of clusters in a data set. J Stat Softw 2014:61:1-36.

31 Zimmerman JE, Kramer AA, McNair DS, et al. Acute Physiology and Chronic Health Evaluation (APACHE) IV: hospital mortality assessment for today's critically ill patients. Crit Care Med 2006;34:1297-310.

32 Villar J, Ambrós A, Soler J, et al. Age, PaO2 /FIO2, and plateau pressure score: a proposal for a simple outcome score in patients with the acute respiratory distress syndrome. Crit Care Med 2016;44:1361-9.

33 van Buuren S, Groothuis-Oudshoorn K. mice: Multivariate Imputation by Chained Equations in R. J Stat Softw 2011. http://doc.utwente.nl/78938/1/Buuren11mice.pdf (accessed 3 Oct 2014)

34 Ware LB, Koyama T, Billheimer DD, et al. Prognostic and pathogenetic value of combining clinical and biochemical indices in patients with acute lung injury. Chest 2010;137:288-96.

35 Papazian L, Forel JM, Gacouin A, et al. Neuromuscular blockers in early acute respiratory distress syndrome. N Engl I Med 2010;363:1107-16.

36 Agustí A, Antó JM, Auffray C, et al. Personalized respiratory medicine: Exploring the horizon, addressing the issues: Summary of a BRN-AJRCCM workshop held in Barcelona on June 12, 2014. Am J Respir Crit Care Med 2015;191:391-401.

37 Sapru A, Calfee CS, Liu KD, et al. Plasma soluble thrombomodulin levels are associated with mortality in the acute respiratory distress syndrome. Intensive Care Med 2015:41:470-8.

38 Nakamura T, Sato E, Fujiwara N, et al. Increased levels of soluble receptor for advanced glycation end products (sRAGE) and high mobility group box 1 (HMGB1) are associated with death in patients with acute respiratory distress syndrome. Clin Biochem 2011:44:601-4.

39 Matthay MA, Zimmerman GA. Acute lung injury and the acute respiratory distress syndrome: four decades of inquiry into pathogenesis and rational management. $A m$ J Respir Cell Mol Biol 2005:33:319-27.

40 Prescott HC, Calfee CS, Thompson BT, et al. Towards smarter lumping and smarter splitting: rethinking strategies for Sepsis and ARDS Clinical Trial Design. Am J Respir Crit Care Med 2016;194:147-55. 